\title{
EFSUMB CEUS Liver Webinars
}

CHAIR: Paul Sidhu. SPEAKER: Christoph F Dietrich The role of CEUS in diagnosis and patient management of primary liver cancer (HCC). Bern, Switzerland

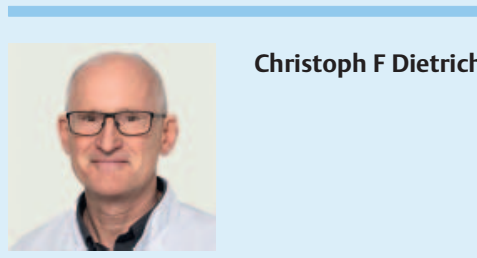

Learning objectives

- The patient at risk

- Screening recommendations

- CEUS criteria of malignancy in liver cirrhosis

- How to perform CEUS

- Liver Imaging Reporting and Data System (LI-RADS), CEUS, CECT, CEMRI

- The frequency (and signs) of benign FLL in liver cirrhosis

- Treatment options
SPEAKER: Mikael Sawatzki. The role of CEUS in diagnosis and patient management of liver metastases. St. Gallen, Switzerland

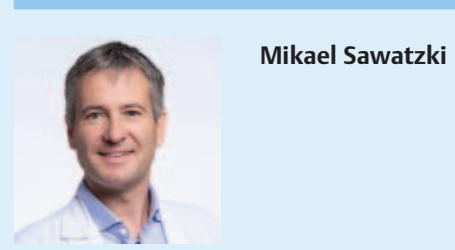

Liver metastases are the most common malignant liver lesions in a non-cirrhotic liver. On abdominal ultrasound, liver metastases may present as hypoechoic, isoechoic or hyperechoic liver lesions, and differentiation from benign lesions (e. g., hyperechoic hemangiomas versus liver metastases) is not always possible. Contrast-enhanced ultrasound (CEUS) has a high diagnostic accuracy in the detection and differentiation of focal liver lesions. CEUS has also been shown to detect liver metastases missed by CT.

Learning objectives include:

- CEUS criteria of malignancy

- Examples of CEUS in various types of liver metastases

- Current study results of CEUS for the detection of colorectal liver metastases

- Recommendation of which patient groups may benefit from additional CEUS
SPEAKER: Eddie Leen. The role of CEUS for planning, guidance, monitoring and follow-up of focal ablation therapies in liver cancer. London, UK

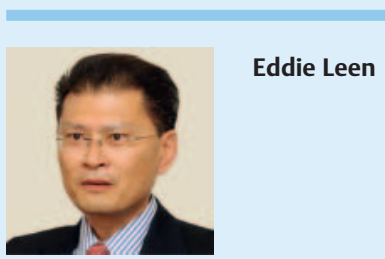

Learn about new application of ultrasound in guided interventional procedures: liver malignancy biopsy, primary and secondary liver malignancy ablation. Understand consolidated and new indications to ultrasound-guided interventional procedures: PEI, RFA, MWA, IRE.

Appreciate the advantages and disadvantages of ultrasound-guided interventional procedures from consolidated to new applications: analysis of data from personal experiences and literature series comparison and discussion.

Become familiar with the new techniques of ultrasound guidance and with the new diagnostic and therapeutic indications for ultrasound-guided interventional procedures: CEUS imaging, fusion imaging, elastosonographic imaging for liver malignancy biopsy and treatment, MWA, IRE, combination treatment.

Thursday 20 May 2021 17.00 CEST

Find the webinar registration link on the EFSUMB website

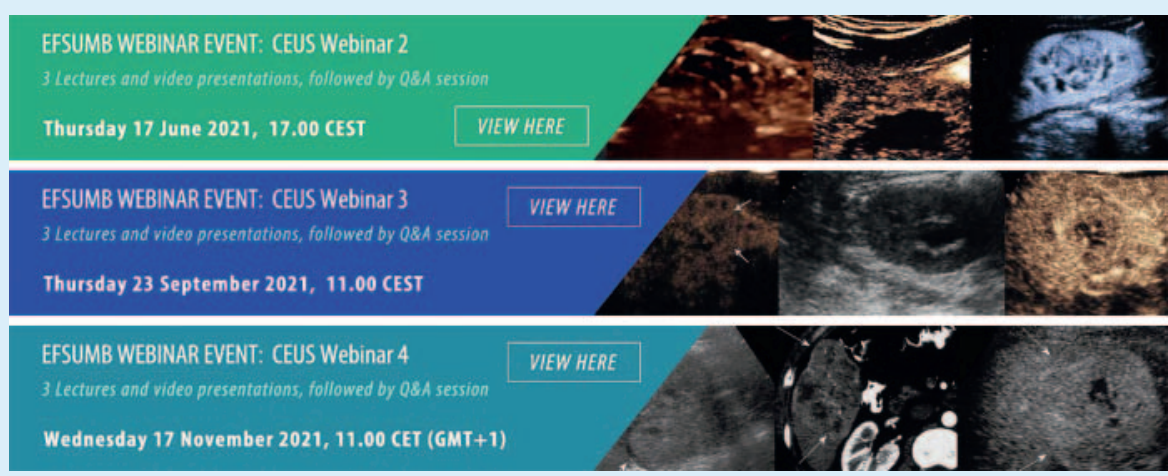

